\title{
EDITORIAL
}

\section{Should we abandon standard forceps biopsy to diagnose an endobronchial lesion?}

\author{
C. Dooms
}

$\mathbf{T}$ he diagnostic yield of flexible bronchoscopy with direct standard forceps biopsy ranges from $72 \%$ to $82 \%$ in large (>200 patients) case series of patients with endobronchial visible tumours presenting as an exophytic mass or submucosal lesion [1]. At least three endobronchial forceps biopsy samples are recommended at the risk of mild self-limiting $(10 \%)$ or severe life-threatening $(<0.02 \%)$ bleeding [2]. The addition of endobronchial needle aspiration to endobronchial forceps biopsy at first bronchoscopy or a repeat flexible bronchoscopy with endobronchial forceps biopsy increases the diagnostic yield to $>90 \%[3,4]$.

A flexible cryoprobe (ERBE USA, Inc., Marietta, GA, USA) for use within a flexible bronchoscope was introduced in 1994. Shortly thereafter, MATHUR et al. [5] and VERGNON et al. [6] described its application in the treatment of malignant airway obstruction (palliation) and radio-occult early-stage lung cancer (cure) applying multiple freeze/thaw cycles of $\sim 30 \mathrm{~s}$. More than a decade later, the role of cryotherapy still remains limited compared with other endobronchial treatment techniques. In 2008, HETZEL et al. [7] stated the feasibility (sample size and tissue quality) of flexible cryoprobes to harvest tissue for histological diagnosis of exophytic lesions during flexible bronchoscopy. In 2010, the same authors reported a diagnostic yield of $90 \%$ for this cryobiopsy technique in 296 endobronchial lesions, and a complication rate of moderate/severe bleeding in $1.3 \%$ requiring local treatment (argon plasma coagulation or bronchus blocker) or additional systemic treatment [8]. It is not the absolute freezing temperature but the sticking property of the probe tip to the tissue that is responsible for the size of the tissue sample. This sticking property depends on tissue type (such as perfusion), probe tip diameter, and the application pressure and time (usually 2-3 s).

In this issue of the European Respiratory Journal, HETzEL et al. [9] present the results of a multi-centre, randomised trial comparing cryobiopsy to endobronchial forceps biopsy during flexible bronchoscopy to obtain a histopathological diagnosis of suspected endobronchial malignant lesions. Biopsies were obtained with $2.4-\mathrm{mm}$ diameter cryoprobes or $2.6-\mathrm{mm}$ ( $2.0 \mathrm{~mm}$ in $27 \%$ ) claw forceps biopsies. The diagnostic yield and number of biopsies taken were significantly higher for the cryobiopsy technique than the endobronchial forceps biopsy ( $95 \%$ versus $85 \%(\mathrm{p}<0.001)$ and 3.5 versus $3.2(\mathrm{p}<0.001)$, respectively). The larger number of cryobiopsies taken combined with the fact that the diameter of a sample taken by cryoprobe technique is twice

CORRESPONDENCE: C. Dooms, Dept of Pulmonology, University Hospitals Leuven, Herestraat 49 B-3000 Leuven, Belgium. E-mail: christophe.dooms@uzleuven.be the size/diameter of forceps biopsies, might account for the higher diagnostic yield obtained with cryobiopsies. Although a diagnostic yield of $95 \%$ is excellent, there is a two-fold disadvantage to performing the cryobiopsy technique under local anaesthesia. First, a self-limiting, mild bleeding rate of $62 \%$ for cryobiopsies, as well as a severe bleeding rate requiring argon plasma coagulation thermocoagulation in $4 \%$ of cryobiopsies versus $0.7 \%$ of forceps biopsies, might be worrisome to many of the pulmonologists performing bronchoscopies. Secondly, the cryobiopsy technique requires intubation under mild sedation with an endotrachial tube. As the cryobiopsies are larger than the working channel of a flexible bronchoscope, the latter requires the ability to be easily removed from and reinserted into the airways. This might also be worrisome to many of the pulmonologists performing bronchoscopies.

It is clear that the cryobiopsy technique extends the armamentarium of our diagnostic techniques, but given the good performance characteristics of traditional forceps biopsies to obtain a histopathological diagnosis and the concerns raised earlier, it would not seem that the routine use of cryobiopsies as a single diagnostic technique is warranted today. However, immunohistochemical and molecular pathology techniques are increasingly applied to biopsy specimens obtained via bronchoscopy for an individualised diagnostic and therapeutic approach to metastatic lung cancer patients $[10,11]$. The importance of accurate histological subtyping of nonsmall cell lung cancer and the expanding field of lung cancer genotyping has an increasing impact on pathology and clinical practice. Small endobronchial biopsies obtained by forceps biopsy techniques might be insufficient to answer these needs. An adequate quantity of DNA is usually provided by between five to $105-\mu \mathrm{m}$ sections of the tumour biopsy [12]. With the aim of collecting sufficient tissue, a higher number of forceps biopsies (four to five instead of one to two) should be obtained. Thus, the larger samples sizes and higher tissue quality claimed by cryobiopsies might complement routine clinical practice in the near future for that purpose. However, more translational research is needed in this domain to justify cryobiopsies as part of routine flexible bronchoscopy.

\section{STATEMENT OF INTEREST}

None declared.

\section{REFERENCES}

1 Rivera MP, Mehta AC, American College of Chest Physicians. Initial diagnosis of lung cancer: ACCP evidence-based clinical 
practice guidelines (2nd edition). Chest 2007; 132: Suppl. 3, 131s-148s.

2 Shure D, Astarita R. Bronchogenic carcinoma presenting as an endobronchial mass. Chest 1983; 83: 865-867.

3 Dobler C, Crawford A. Bronchoscopic diagnosis of endoscopically visible lung malignancies: should cytologic examinations be performed routinely? Intern Med J 2009; 39: 806-811.

4 Govert J, Dodd L, Kussin P, et al. A prospective comparison of fiberoptic transbronchial needle aspiration and bronchial biopsy for bronchoscopically visible lung carcinoma. Cancer 1999; 87: 129-134.

5 Mathur PN, Wolf KM, Busk MF, et al. Fiberoptic bronchoscopic cryotherapy in the management of tracheobronchial obstruction. Chest 1996; 110: 718-723.

6 Vergnon JM, Huner RM, Moghissi. Place of cryotherapy, brachytherapy and photodynamic therapy in therapeutic bronchoscopy of lung cancers. Eur Respir J 2006; 28: 200-218.

7 Hetzel J, Hetzel M, Hasel C, et al. Old meets modern: the use of traditional cryoprobes in the age of molecular biology. Respiration 2008; 76: 193-197.
8 Schumann C, Hetzel J, Babiak A, et al. Cryoprobe biopsy increases the diagnostic yield of endobronchial tumor lesions. J Thorac Cardiovasc Surg 2010; 140: 417-421.

9 Hetzel J, Eberhardt R, Herth FJF, et al. Cryobiopsy increases the diagnostic yield of endobronchial biopsy: a multicentre trial. Eur Respir J 2012; 39: 685-690.

10 Scagliotti G, Parikh P, von Pawel J, et al. Phase III study comparing cisplatin plus gemcitabine with cisplatin plus pemetrexed in chemotherapy-naive patients with advanced-stage non-small-cell lung cancer. J Clin Oncol 2008; 26: 3543-3551.

11 Fukuoka $\mathrm{M}, \mathrm{Wu} \mathrm{Y}$, Thongprasert $\mathrm{S}$, et al. Biomarker analyses and final overall survival results from a phase III, randomized, openlabel, first-line study of gefitinib versus carboplatin/paclitaxel in clinically selected patients with advanced non-small-cell lung cancer in Asia (IPASS). J Clin Oncol 2011; 29: 2866-2874.

12 Gridelli C, De Marinis F, De Maio M, et al. Gefitinib as first-line treatment for patients with advanced non-small-cell lung cancer with activating epidermal growth factor receptor mutation: implications for clinical practice and open issues. Lung Cancer 2011; 72: 3-8. 